This item was submitted to Loughborough's Research Repository by the author.

Items in Figshare are protected by copyright, with all rights reserved, unless otherwise indicated.

\title{
Multiplex measurement of diffusion in zinc battery electrolytes from microfluidics using Raman microspectroscopy
}

PLEASE CITE THE PUBLISHED VERSION

https://doi.org/10.1016/j.apenergy.2020.115687

PUBLISHER

Elsevier

VERSION

AM (Accepted Manuscript)

PUBLISHER STATEMENT

This paper was accepted for publication in the journal Applied Energy and the definitive published version is available at https://doi.org/10.1016/j.apenergy.2020.115687

LICENCE

CC BY-NC-ND 4.0

\section{REPOSITORY RECORD}

Chen, Bin Bin, Jin Xuan, Gregory James Offer, and Huizhi Wang. 2020. "Multiplex Measurement of Diffusion in Zinc Battery Electrolytes from Microfluidics Using Raman Microspectroscopy". Loughborough University. https://hdl.handle.net/2134/13095620.v1. 


\title{
Multiplex measurement of diffusion in zinc battery electrolytes from
}

\section{microfluidics using Raman microspectroscopy ${ }^{1}$}

\author{
Binbin Chen ${ }^{1}$, Jin Xuan ${ }^{2}$, Gregory James Offer ${ }^{1}$, Huizhi Wang ${ }^{1 *}$ \\ ${ }^{1}$ Department of Mechanical Engineering, Imperial College London, United Kingdom \\ ${ }^{2}$ Department of Chemical Engineering, Loughborough University, Loughborough, \\ United Kingdom \\ *Corresponding author. huizhi.wang@imperial.ac.uk (H. Wang)
}

\begin{abstract}
Rechargeable zinc batteries have emerged as an inexpensive and safe post-lithium-ion battery technology and have received increasing research interest. Developing suitable electrolytes and understanding their transport properties lie at the heart of successful zinc battery technologies as the battery behaviour is a strong function of ion transport in the electrolytes. To accelerate the research and development process, herein we demonstrate a low-cost and high-throughput approach to measure the diffusion in zinc electrolytes at different concentrations simultaneously. The new approach combines Raman microspectroscopy and a multiplexed microfluidic chip with integrated micromixers, concentration gradient generators and a Y-sensor array. Aqueous-based zinc sulphate electrolytes, widely used in zinc batteries, were used for a proof-ofconcept. The measured diffusion coefficients for different electrolyte concentrations show good agreement with literature values. With four electrolyte samples in this study, the developed approach requires minimum $0.5 \mathrm{~mL}$ of the electrolyte solutions and 30 mins, which is over ten times faster than a typical diffusion measurement with the conventional electrochemical approach in restricted-diffusion cells. The
\end{abstract}

\footnotetext{
${ }^{1}$ The short version of the paper was presented at ICAE2019, Aug 12-15, Västerås, Sweden. This paper is a substantial extension of the short version of the conference paper.
} 
microfluidic chip is readily scalable to further increase the throughput, and can be extended to for use of measuring different (i.e. organic and aqueous) and even mixtures of electrolytes (i.e. ethylene carbonate and dimethyl carbonate) as well as salts $\left(\mathrm{Li}^{+}, \mathrm{Na}^{+}, \mathrm{Mg}^{2+}\right.$, etc. $)$.

Keywords: Zinc battery; Electrolyte; Diffusion; Microfluidics; Raman microspectroscopy. 


\section{Introduction}

Energy storage has become one of the most important topics in the global transition to a renewable energy economy. In this regard, electrochemical storage technologies such as batteries and redox flow cells have been recognized as the most promising technologies for portable electronics, electric vehicles and backup power supplies.[1] Though lithium-ion batteries are the current dominant technology, their inherent drawbacks associated with safety and cost have triggered efforts in researching safer and lower-cost alternative to lithium-ion batteries.[2] Rechargeable zinc ion batteries , consisting of a zinc anode and a zinc intercalating cathode in a zinc-salt-containing electrolyte and using zinc ions as charge carriers, have emerged as a promising postlithium-ion option.[3, 4] So far, zinc ion batteries have been applied for powering wildlife equipment as well as small electronics such as hearing aids.[5] The electrolyte transporting zinc ions between the anode and the cathode plays a key role in determining the battery behaviour.[6] For example, dendrite formation of the zinc anode, a major bottleneck for zinc batteries, is known to happen under diffusionlimited conditions.[7] As a consequence, accurate measurements of diffusion properties are essential for numerical simulations to accurately reproduce the behaviour of battery devices. In this regard, an effective and high-throughput method for measuring the diffusion in the electrolytes would be very useful for characterising electrolytes and screening suitable electrolyte formulations for improved performance.

Over the decades, researchers have been developing and improving techniques to determine diffusion coefficients based on very different principles. Work on transport or thermodynamic characterization of species abounds in the literature, but they are time consuming and laborious. Conventional methods evaluate the diffusion 
coefficient by measuring the concentration of the diffusing species as a function of the depth of penetration. Typical measurements last from an hour up to several days for each test, and consume relatively large sample volumes ranging from several $\mathrm{mL}$ to hundreds of mL.[8] Pulsed-field gradient spin echo enabled the possibility of determination of self-diffusion coefficients using nuclear magnetic resonance, but this is very expensive.[9] In contrast, electrochemical methods based on chronoamperometry in solutions provide more affordable and easily operated methods to measure diffusion coefficients in solutions.[10, 11] However, the biggest limitation of these electrochemical methods is that the species have to be well chosen to fit the system.[12] There has been lack of an efficient and low-cost technique for measuring the diffusion in electrolytes for electrochemical energy systems.

Microfluidics is particularly suited to overcome the main limitations in the measurement of diffusion coefficients as the small dimensions result in short measurement time since the diffusion time is proportional to the square of diffusion distance.[13] Furthermore, microfluidic systems allow for significant reductions in the sample volumes required due to the small channel volumes. The mechanism of mixing fluid streams in a microscale channel having dimensions of several hundred microns and below is quite different from that in a conventional macroscale channel.[14] The utilization of laminar flow features in the microchannels enables numerous functionalities, such as sample preparation, concentration gradient generation, molecular interactions and transport assay.[15, 16] In the simplest configuration, the channel introduces two pressure-driven fluid streams side by side. As convective mixing is absent due to the small Reynolds numbers $(\operatorname{Re}<1)$, mixing at the microscale occurs primarily via molecular diffusion at the interface between two streams.[17] The techniques for visualising the concentration profiles in 
microfluidic systems have been widely reported in the fields of biology and chemistry. For optically active or fluorescent species, microfluidics devices coupled with fluorescence detection have been developed to measure the concentration distributions.[18, 19] While for optically inactive species, Raman micro-spectroscopy provides an opportunity to eliminate the use of fluorescent indicators.[20] Raman spectroscopy is a nonintrusive detection technique that allows for measurement of the local concentration of species of interest with high accuracy and spatial resolution,[21] which has been regarded as a particularly suitable analytical tool to detect optically inactive species. Very recently, this approach has been employed for mapping $\mathrm{Li}^{+}$ distributions for Li-ion batteries.[22]

In this study, we demonstrate a new microfluidic approach to measure the diffusion in zinc electrolytes at different concentrations. The approach combines Raman microspectroscopy and a multiplexed microfluidic chip with integrated micromixers, concentration gradient generators and a Y-sensor array to enable effective and highthroughput diffusion measurements. As a proof of concept, diffusion in aqueousbased zinc sulphate $\left(\mathrm{ZnSO}_{4}\right)$ electrolytes at different concentrations were measured and compared with literature data.

\section{Experimental}

\subsection{Concentration gradient generation}

Figure 1 shows the microfluidic concentration gradient generator (CGG) designed for automatically generating electrolyte samples at different concentrations. The design follows the tree-shape configuration which is commonly used in microfluidics.[23, 24] As detailed in Figure 1 (a2), in the CGG, streams are split at each bifurcation and flow into the neighbouring branch channels of the next tier with defined ratios. Thereafter, 
two neighbouring branch streams of middle confluences are combined to form new concentrations. The concentrations of the two outermost branch streams remain the same till the last stage. After repeated splitting, combining and mixing at each tier, intermediate concentrations are gradually generated. The splitting ratio is a key parameter during the process. The theoretical flow rates and concentration distribution can be calculated by integrating the splitting ratio with initial source concentrations. In a typical way for a pressure-driven system with similar configurations of each subchannels, linear concentration gradients can be obtained.

The CGG was fabricated by laser cutting and engraving with a carbon dioxide laser ablation system (VLS 2.30, Universal Laser System Inc., USA). It consisted of three polymethyl methacrylate (PMMA) layers. The middle layer with a thickness of $3 \mathrm{~mm}$ was engraved with a 2-tier distribution tree-shape network on both top and bottom surfaces. The engraving setting parameters used were $100 \%$ of power and $20 \%$ of speed, obtaining branch channels of $300 \mu \mathrm{m}$ wide and $195 \mathrm{~mm}$ long. The top layer with a thickness of $250 \mu \mathrm{m}$ sealed with four inlets and eight outlets for fluidic access. Each of the networks occupied two inlets and four outlets access. The bottom layer was a rectangular plate used for encapsulation. Three layers were sealed by doublesided tape with each other. Tubing was then bonded to the holes on the top layer to make the fluidics connections.

To verify the design of CGG, two series of $\mathrm{ZnSO}_{4}$ solutions with different concentrations (Series 1: $0 \mathrm{M}$ with 0.9 M into bottom network, and Series 2: $0.1 \mathrm{M}$ with $1 \mathrm{M}$ into top network; $\sim 10 \mathrm{~mL}$ ) were pumped into the CGG at different flow rates $(/ P L Q a / P L Q) \quad$. The volume of the solution at every single outlet was measured to represent the flow rate of the outlet. The concentrations were then investigated by Raman spectroscopy. 


\subsection{Y-sensor}

A Y-sensor was employed for the diffusion measurements (Figure 1 (a3)). An array of Y-sensors was cut by the laser cutting machine on a piece of double-sided tape. The sensors are aligned in parallel with a distance of $18 \mathrm{~mm}$ between each other. Four short lines were marked nearby the channels to locate the position $(\mathbf{L}=25 \mathrm{~mm})$ for Raman inspection. The channels were then sandwiched by two thin glass plates of 0.1 PPW K LFN 7K HJ ODV V GR-XEEGHW WSJODV V ODIHUV ZHUHDW WDFK HG WR HDFK RWK HUGL The width of channels was determined by the confocal Raman microscope (inVia 4RQWRU FRQIRFDO 5DPDQ PLFURVFRSH 5HQLVKDZ 8. DIWHU VHDOLQJ ìP The thickness was also determined by recording the difference in microscope position ZKHQWKHIRFXVHVZHUHRQWKHWRSDQGERWW RPJODVVHOHFWUROIWHLQWHUIDFH The cross sectional dimensions of the Y-sensor was designed to give an aspect ratio of 12. The flow rates employed in this study ZHUHORZHUWKDQ/PLQWRHQVIXoby enough residence time. Under this condition, the time needed for depth-wise diffusion is much less than the width-wise diffusion and axial convection, minimising the "butterfly effect",[25] in which diffusion broadening regions are formed near the top and bottom walls due to the parabolic velocity profile across the channel. The normalized concentration distribution at the downstream of the channel can be expressed as:[20]

$$
C_{0}=\frac{1}{2}\left(1+\operatorname{erf} \frac{y}{2 \sqrt{D t}}\right)
$$

where $C_{0}$ is the normalized concentration difference, $D$ is diffusion coefficient, $t$ is average residence time and $y$ is the distance from the middle interface. Once the concentration profile is measured, the diffusion coefficient can be calculated from the above equation. 


\subsection{Multiplexed chip design and operation for Raman inspection}

The multiplexed microfluidic chip was assembled by integrating the CGG and the array of Y sensors. The sub-branches of four Y-shape channels were connected to the four pairs of the CGG outlets for the access of solutions. Figure 1 (a1) shows the experimental setup and Figure 1 (b) shows the photo of the multiplexed chip developed in the present study. All the tests were conducted under room temperature (i.e., $25^{\circ} \mathrm{C}$ ). The inspection of solution concentrations in the Y-shape channels was performed using the Raman microscope. The signal was illuminated by radiation from an argon-LRQODVHU QP\$x objective was used for all measurements. Care was taken to record spectra in the middle plane of the channels by focusing the laser spot on the glass-electrolyte interface at the top of the channel, recording the $\mathrm{z}$ position, then focusing the laser spot on the interface at the bottom of the channel, recording the position, and then adjusting the focal plane of the microscope to be halfway between these two positions.

The calibration tests were conducted within a single Y-shape channel structure. To ensure no effect from cell structure on the Raman signal, a background Raman signal with a focus on glass and mid-plane of the channel was first measured. Thereafter, Raman signals of solutions with various concentrations were measured by injecting different solutions into the channel. Thereafter, the relationship between the concentration and Raman intensity was established.

For concentration distribution inspection, the desired position $(\mathbf{L}=25 \mathrm{~mm})$ for inspection was located and then brought into focus on the middle plane. To further eliminate the wall effects on the ion diffusion, only central $1.2 \mathrm{~mm}$ width was inspected. Raman spectra were recorded at each point every $20 \mathrm{~m}$ along the diffusion 
direction. Acquisition parameters were 1 second exposure and accumulated 5 times for each point, resulting in the whole acquisition time of an image of around 6 minutes. Then the object stage was moved to bring the next channel for Raman inspection. The local concentration distribution in each channel could be probed from the intensities of the Raman bands at a specific shift position. Concentration maps in four channels were then obtained.

\subsection{Chemical reagents and flow control}

$\mathrm{ZnSO}_{4}$ aqueous solutions with different concentrations were prepared by dissolving $\mathrm{ZnSO}_{4} \cdot 6 \mathrm{H}_{2} \mathrm{O}$ powder $(>99.6 \%, \mathrm{VWR}, \mathrm{UK})$ in deionized water $(0 \quad$ Barnstead, NANOpure Diamond, USA). The solutions were pumped into the system by a syringe pump (LSP02-1B, LongerPump, China) at controllable flow rates.

\section{Results and discussion}

\subsection{Calibration}

Figure 2 (a) shows the background Raman spectra measured on a blank channel with a focus on the glass layer and mid-plane of the channel. Although the glass layer has a clear Raman spectrum, it was well averaged over the height to a low level with a focus on middle plane of the channel, especially around the shift position of $981 \mathrm{~cm}^{-1}$, corresponding to the bandshape of $v_{1}-\mathrm{SO}_{4}^{2-}$.[26] Figures 2 (b) and (c) summarize the results obtained for the calibration to establish the relationship between the local concentrations of the $\mathrm{ZnSO}_{4}$ and the intensities of Raman bands. Figure 2 (b) shows the Raman spectra of the region between 940 to $1000 \mathrm{~cm}^{-1}$ with the concentration of $\mathrm{ZnSO}_{4}$ increasing from $0 \mathrm{M}$ to $1 \mathrm{M}$. The peak intensities at the shift position of 981 $\mathrm{cm}^{-1}$ increase with the increase of concentration. A well-fit linear relationship can be 
obtained between $\mathrm{ZnSO}_{4}$ concentration and the Raman sideband intensities on the position of $981 \mathrm{~cm}^{-1}$ as shown in Figure 2 (c). This linear relationship allows us to use the relative intensities directly to represent the local concentrations of $\mathrm{SO}_{4}^{2-}$ in the channels.

\subsection{Flow rate and concentration distributions in CGG}

The fluidic network in CGG can be considered as analogous to an electrical circuit, where the pressure drop $\mathbf{P}$, volumetric flow rate $\mathbf{Q}$ and hydraulic resistance $\mathbf{R}$ correspond to a voltage drop, current and electric resistance, respectively.[27, 28] Analogous to Ohm's law, the Hagen-Poiseuille's law $\boldsymbol{P}=\boldsymbol{Q R}$ can be deduced. Therefore, the splitting ratio and mixing behaviour can be resolved using electrical circuit theory. In the channels of first tier shown in Figure 1 (a2), the following functional relationships can be established.

$Q_{1}=Q_{3}+Q_{4}$

$$
\begin{gathered}
Q_{2}=Q_{5}+Q_{6} \\
\Delta P_{1}=Q_{3} R_{1} \\
\Delta P_{2}=\left(Q_{3}+Q_{4}\right) R_{2} \\
\Delta P_{3}=Q_{5} R_{3} \\
\Delta P_{1}=\Delta P_{2}=\Delta P_{3}
\end{gathered}
$$

The flow rate through each branch channel can be calculated as follows

$$
\begin{gathered}
Q_{3}=\frac{Q_{1} R_{2} R_{3}+Q_{2} R_{2} R_{3}}{R_{1} R_{2}+R_{1} R_{3}+R_{2} R_{3}} \\
Q_{4}+Q_{5}=\frac{Q_{1} R_{1} R_{3}+Q_{2} R_{1} R_{3}}{R_{1} R_{2}+R_{1} R_{3}+R_{2} R_{3}}
\end{gathered}
$$


$Q_{6}=\frac{Q_{1} R_{1} R_{2}+Q_{2} R_{1} R_{2}}{R_{1} R_{2}+R_{1} R_{3}+R_{2} R_{3}}$

Assuming the two inlets are equal, i.e. $\boldsymbol{Q}_{1}=\boldsymbol{Q}_{2}=\boldsymbol{q}$. With the same channel configuration of branch streams, hydraulic resistances are considered to be the same $\left(\boldsymbol{R}_{1}=\boldsymbol{R}_{2}=\boldsymbol{R}_{3}=\boldsymbol{r}\right)$. Therefore, the flow rates at each outlet are expected to equal to each other:

$Q_{3}=Q_{4}+Q_{5}=Q_{6}=\frac{2}{3} q$

Following the same deduction process, the flow rates at the outlets of the second tier are the same $(\boldsymbol{q} / \mathbf{2})$. With the same flow rate in each channel, a linear concentration gradient should be obtained after the streams pass through the CGG.

Two series of $\mathrm{ZnSO}_{4}$ solutions ( $0 \mathrm{M}$ vs. $0.9 \mathrm{M}$ and $0.1 \mathrm{M}$ vs. $\left.1.0 \mathrm{M}\right)$ were injected into the top and bottom networks of the CGG, respectively, at rates ranging from 2.5 /PLQ - /PLQ . Figure 3 (a) verifies the uniformity of flow rates at all outlets. By using the volume of the first outlet as a benchmark, the difference between flow rates at four outlets was lower than $0.5 \%$. The result ensured the streams into the Yshape channels were symmetric.

The concentration of $\mathrm{ZnSO}_{4}$ in the outlet solution was then detected by Raman spectroscope. A linear increase in the Raman intensities at shift position of $981 \mathrm{~cm}^{-1}$ of liquids from four outlets was observed for both networks of the CGG as shown in Figure 3 (b). The increase matched well with the linear fitting in Figure 2 (c), resulting in four pairs of $\mathrm{ZnSO}_{4}$ solutions: $0 \mathrm{M}$ vs. $0.1 \mathrm{M}, 0.3 \mathrm{M}$ vs. $0.4 \mathrm{M}, 0.6 \mathrm{M}$ vs $0.7 \mathrm{M}$, and $0.9 \mathrm{M}$ vs $1.0 \mathrm{M}$ for the four $\mathrm{Y}$-shape channels. The constant difference between two adjacent flow streams minimizes the effects of large concentration differences. In the Y-shape channel, as the two input streams meet and flow down the channel, ions diffused from the higher side to the lower side resulting in a concentration distribution along the width of the channel. 


\subsection{Diffusion coefficient of $\mathrm{ZnSO}_{4}$}

The functionality of the integrated multiplex-assay system was then verified by the concentration distribution in the channel and the fitting result with the data from the literature. Tests at three different flow rates of 2.5, 10 and 25/ PLQ were conducted. The effect of decreasing the flow rate was to increase the average residence time and thereby increase the extent of internal diffusion of $\mathrm{ZnSO}_{4}$ at the fixed observation location. Raman signal data were captured and normalized by the intensity difference of two flow streams. Figure 4 shows the normalized concentration profiles obtained at the downstream of the four channels. The experimental results fit well with the profiles yielded by Equation (1) and the diffusion coefficients of $\mathrm{ZnSO}_{4}$ with corresponding concentrations from literature $\left(0.1 \mathrm{M}: 5.69 \times 10^{-10} \mathrm{~m}^{2} \mathrm{~V} 0 \quad: 4.63 \times\right.$ $10^{-10} \mathrm{~m}^{2} \mathrm{~V} 0$ ó $\quad{ }^{-10} \mathrm{~m}^{2} \mathrm{~V} 0$ ó $\quad{ }^{-10} \mathrm{~m}^{2} \mathrm{~V} \cdot[29,30]$ With all the results obtained, the multiplex-assay functionality of the system has been proven that it can generate accurate concentration profiles in the channel array simultaneously, which can be further used to represent the diffusion coefficients of ions in solutions with different concentrations.

\subsection{Throughput and assay time}

The newly developed microfluidic system achieves a multiplex assay for ion diffusion coefficient measurement in one single system for the first time. A dual network CGG provides even flow rates and linear concentration gradients for the outlets. It provides constant concentration differences for the Y-shape channels, which ensures that a good accuracy of diffusion in the microfluidic channel can be achieved with this system. During the WHVW ORZIORZUDWHVIURP/PLQWR/PLQZHUHHPSOR IHG 
The Raman measurement for each channel requires approximately 6 minutes. The overall experiment for the whole array could be finished within half an hour, which consumes less than $1 \mathrm{~mL}$ of electrolyte. The electrolyte consumption can be further reduced by reducing the channel size, which is achievable with higher-precision fabrication techniques. Though the current proof-of-concept design contains only 4 channels, the microfluidic chip is readily scalable to increase the number of channels, thereby further increasing the throughput. The same system is applicable for measuring the diffusion in other electrolytes for other electrochemical devices.

\section{Conclusions}

This work reports a multiplexed microfluidic approach for rapid and simultaneous measurement of diffusion coefficients at different concentrations for zinc battery electrolytes for the first time. The new approach combines Raman microspectroscopy and a multiplexed microfluidic chip with integrated micromixers, concentration gradient generators and a Y-sensor array. Aqueous-based zinc sulphate electrolytes, widely used in zinc batteries, were used for a proof-of-concept. The measured diffusion coefficients for different electrolyte concentrations show good agreement with literature values. With four electrolyte samples in this study, the developed approach requires $0.5 \mathrm{~mL}$ of the electrolyte solutions and 30 minutes, which is over ten times faster than a typical diffusion measurement with the conventional electrochemical approach in restricted-diffusion cells. This approach provides a useful tool for screening and optimising electrolyte for batteries.

\section{Acknowledgements}


The research is supported by the UK Engineering and Physical Sciences Research \&RXQFLO(365\&YLDJUDQWQXPEHU(36

\section{References}

1. Khaligh, A. and Z. Li, Battery, ultracapacitor, fuel cell, and hybrid energy storage systems for electric, hybrid electric, fuel cell, and plug-in hybrid electric vehicles: State of the art. IEEE transactions on Vehicular Technology, 2010. 59(6): p. 28062814.

2. Hammond, G.P. and T. Hazeldine, Indicative energy technology assessment of advanced rechargeable batteries. Applied Energy, 2015. 138: p. 559-571.

3. Wang, F., O. Borodin, T. Gao, X. Fan, W. Sun, F. Han, ... C. Wang, Highly reversible zinc metal anode for aqueous batteries. Nature materials, 2018. 17(6): p. 543-549.

4. Kundu, D., B.D. Adams, V. Duffort, S.H. Vajargah, and L.F. Nazar, A high-capacity and long-life aqueous rechargeable zinc battery using a metal oxide intercalation cathode. Nature Energy, 2016. 1(10): p. 1-8.

5. Pei, P., K. Wang, and Z. Ma, Technologies for extending zinc-air battery's cyclelife: A review. Applied Energy, 2014. 128: p. 315-324.

6. Han, S.-D., N.N. Rajput, X. Qu, B. Pan, M. He, M.S. Ferrandon, ... A.K. Burrell, Origin of electrochemical, structural, and transport properties in nonaqueous zinc electrolytes. ACS applied materials \& interfaces, 2016. 8(5): p. 3021-3031.

7. Banik, S.J. and R. Akolkar, Suppressing dendritic growth during alkaline zinc electrodeposition using polyethylenimine additive. Electrochimica Acta, 2015. 179: p. 475-481.

8. Hou, T. and C.W. Monroe, Composition-dependent thermodynamic and masstransport characterization of lithium hexafluorophosphate in propylene carbonate. Electrochimica Acta, 2019: p. 135085.

9. Saito, Y., H. Yamamoto, O. Nakamura, H. Kageyama, H. Ishikawa, T. Miyoshi, and M. Matsuoka, Determination of ionic self-diffusion coefficients of lithium electrolytes using the pulsed field gradient NMR. Journal of power sources, 1999. 81: p. 772-776.

10. Moldenhauer, J., M. Meier, and D.W. Paul, Rapid and direct determination of diffusion coefficients using microelectrode arrays. Journal of The Electrochemical Society, 2016. 163(8): p. H672-H678.

11. Saw, E.N., N. Blanc, K. Kanokkanchana, and K. Tschulik, Time-resolved impact electrochemistry-A new method to determine diffusion coefficients of ions in solution. Electrochimica Acta, 2018. 282: p. 317-323.

12. Klett, M., M. Giesecke, A. Nyman, F. Hallberg, R.W. Lindström, G.r. Lindbergh, and I.n. Furó, Quantifying mass transport during polarization in a Li ion battery electrolyte by in situ 7Li NMR imaging. Journal of the American Chemical Society, 2012. 134(36): p. 14654-14657.

13. Berg, O.G. and P.H. von Hippel, Diffusion-controlled macromolecular interactions. Annual review of biophysics and biophysical chemistry, 1985. 14(1): p. 131-158. 
14. Wang, Y., D.Y. Leung, H. Zhang, J. Xuan, and H. Wang, Numerical and experimental comparative study of microfluidic fuel cells with different flow configurations: Coflow vs. counter-flow cell. Applied Energy, 2017. 203: p. 535-548.

15. Gravesen, P., J. Branebjerg, and O.S. Jensen, Microfluidics-a review. Journal of micromechanics and microengineering, 1993. 3(4): p. 168.

16. Nguyen, P., J.W. Carey, H.S. Viswanathan, and M. Porter, Effectiveness of supercritical-CO2 and $\mathrm{N} 2$ huff-and-puff methods of enhanced oil recovery in shale fracture networks using microfluidic experiments. Applied energy, 2018. 230: p. 160174.

17. Shaegh, S.A.M., N.-T. Nguyen, and S.H. Chan, A review on membraneless laminar flow-based fuel cells. International Journal of Hydrogen Energy, 2011. 36(9): p. 56755694.

18. Kamholz, A.E., B.H. Weigl, B.A. Finlayson, and P. Yager, Quantitative analysis of molecular interaction in a microfluidic channel: the T-sensor. Analytical chemistry, 1999. 71(23): p. 5340-5347.

19. Hatch, A., A.E. Kamholz, K.R. Hawkins, M.S. Munson, E.A. Schilling, B.H. Weigl, and P. Yager, A rapid diffusion immunoassay in a T-sensor. Nature biotechnology, 2001. 19(5): p. 461.

20. Salmon, J.-B., A. Ajdari, P. Tabeling, L. Servant, D. Talaga, and M. Joanicot, In situ Raman imaging of interdiffusion in a microchannel. Applied physics letters, 2005. 86(9): p. 094106.

21. Peters, C., L. Wolff, S. Haase, J. Thien, T. Brands, H.-J. Koß, and A. Bardow, Multicomponent diffusion coefficients from microfluidics using Raman microspectroscopy. Lab on a Chip, 2017. 17(16): p. 2768-2776.

22. Forster, J.D., S.J. Harris, and J.J. Urban, Mapping Li+ concentration and transport via in situ confocal Raman microscopy. The journal of physical chemistry letters, 2014. 5(11): p. 2007-2011.

23. Somaweera, H., A. Ibraguimov, and D. Pappas, A review of chemical gradient systems for cell analysis. Analytica chimica acta, 2016. 907: p. 7-17.

24. Wang, Y., T. Mukherjee, and Q. Lin, Systematic modeling of microfluidic concentration gradient generators. Journal of Micromechanics and Microengineering, 2006. 16(10): p. 2128.

25. Song, H., Y. Wang, and K. Pant, Cross-stream diffusion under pressure-driven flow in microchannels with arbitrary aspect ratios: a phase diagram study using a threedimensional analytical model. Microfluidics and nanofluidics, 2012. 12(1-4): p. 265277.

26. Hayes, A.C., P. Kruus, and W.A. Adams, Raman spectroscopic study of aqueous (NH 4) 2 SO 4 and ZnSO 4 solutions. Journal of solution chemistry, 1984. 13(1): p. 61-75.

27. Oh, K.W., K. Lee, B. Ahn, and E.P. Furlani, Design of pressure-driven microfluidic networks using electric circuit analogy. Lab on a Chip, 2012. 12(3): p. 515-545.

28. Lee, K., C. Kim, B. Ahn, R. Panchapakesan, A.R. Full, L. Nordee, . . . K.W. Oh, Generalized serial dilution module for monotonic and arbitrary microfluidic gradient generators. Lab on a Chip, 2009. 9(5): p. 709-717.

29. Albright, J.G. and D.G. Miller, Mutual diffusion coefficients of aqueous ZnSO 4 at $25^{\circ}$ C. Journal of Solution Chemistry, 1975. 4(9): p. 809-816.

30. Awakura, Y., T. Doi, and H. Majima, Determination of the diffusion coefficients of CuSO 4, ZnSO 4, and NiSO 4 in aqueous solution. Metallurgical Transactions B, 1988. 19(1): p. 5-12. 


\section{List of figures}

Figure 1 (a) (a1) Experimental setup and multiplexed microfluidic chip including the design principles and flow distributions in the (a2) CGG unit and (a3) Y-sensors. (b) Photo of the multiplexed microfluidic chip.

Figure 2 (a) Background Raman spectra with a focus on channel glass and midplane of channel. (b) Raman spectra and (c) intensity around the shift position of $981 \mathrm{~cm}^{-1}$ at different $\mathrm{ZnSO}_{4}$ concentrations $(0 \mathrm{M}, 0.05 \mathrm{M}, 0.10 \mathrm{M}, 0.20 \mathrm{M}, 0.30 \mathrm{M}, 0.40 \mathrm{M}, 0.50$ M, 0.60 M, 0.70 M, 0.80 M, 0.90 M, 1.00 M).

Figure 3 (a) Flow rate and (b) concentration distributions at the outlets of the CGG unit.

Figure 4 Normalized concentration profile and fitting concentration distribution within four channels at $\mathbf{L}=25 \mathrm{~mm}$ with flow rates at $25 \mathrm{LPLQ} \quad \mathrm{LPLQDQG}$

LPL QD -d): channel 1-4 with solution concentrations of $0.1 \mathrm{M}, 0.4 \mathrm{M}, 0.7 \mathrm{M}$ and 1.0 M. 
Figure 1

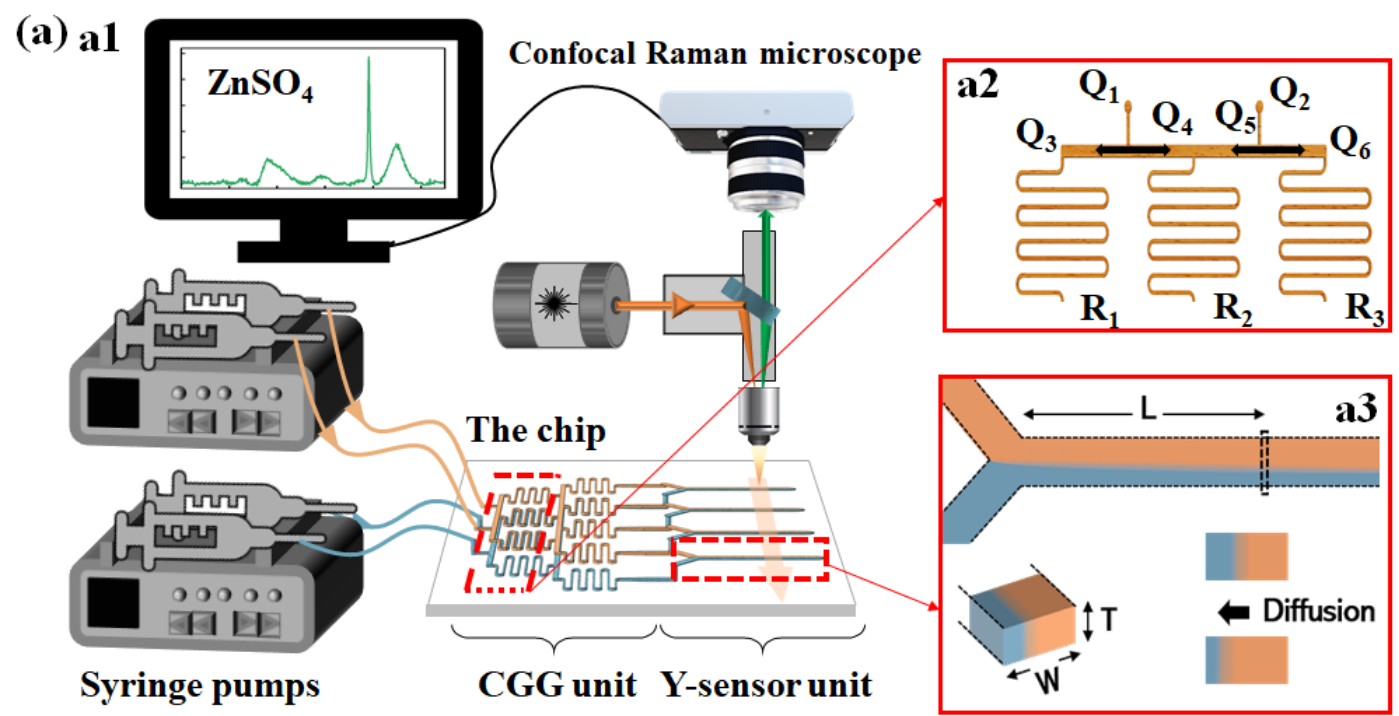

(b)

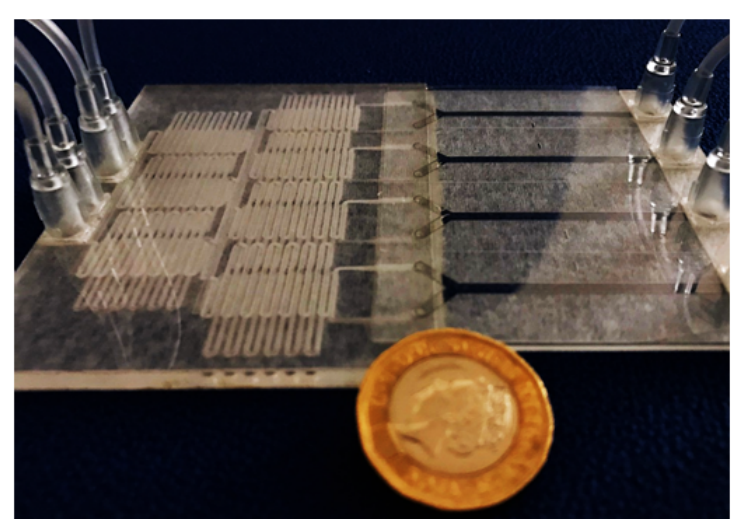


Figure 2
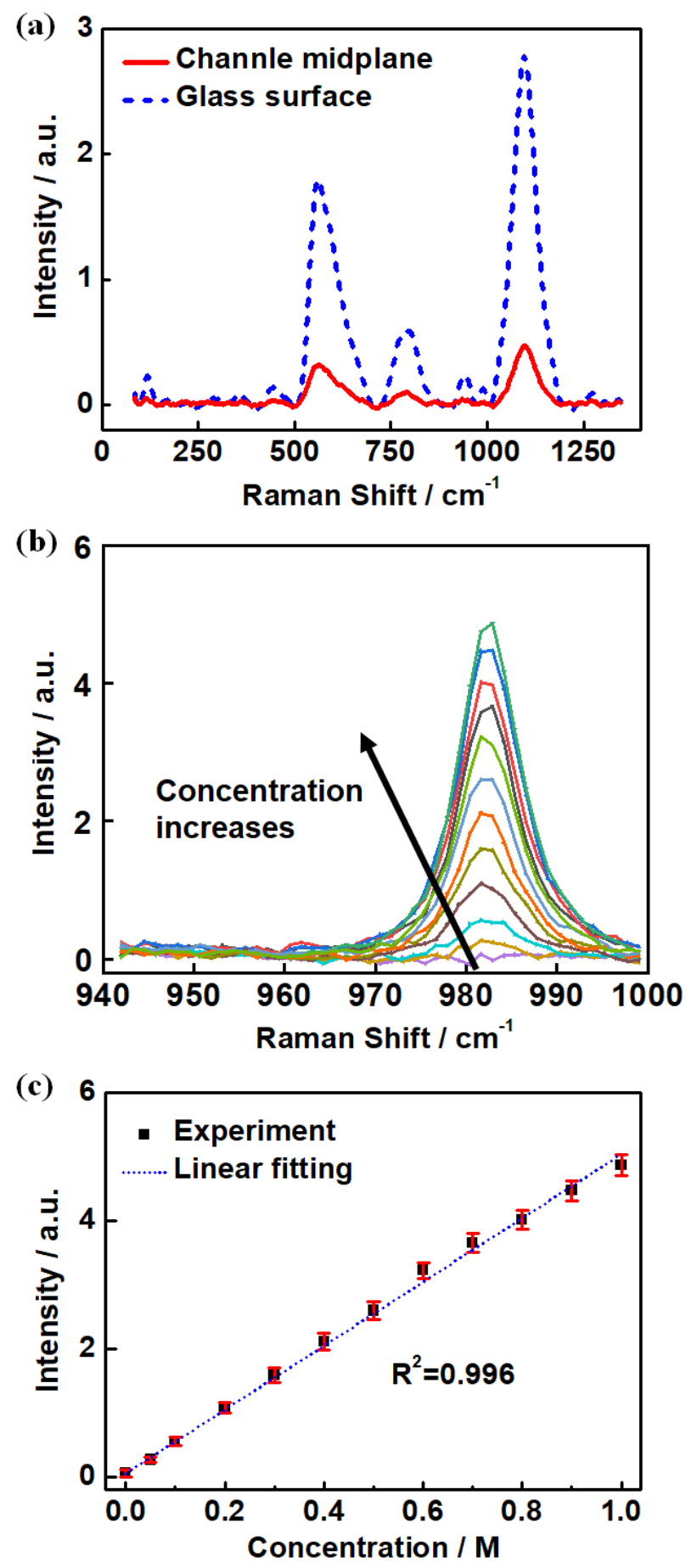
Figure 3
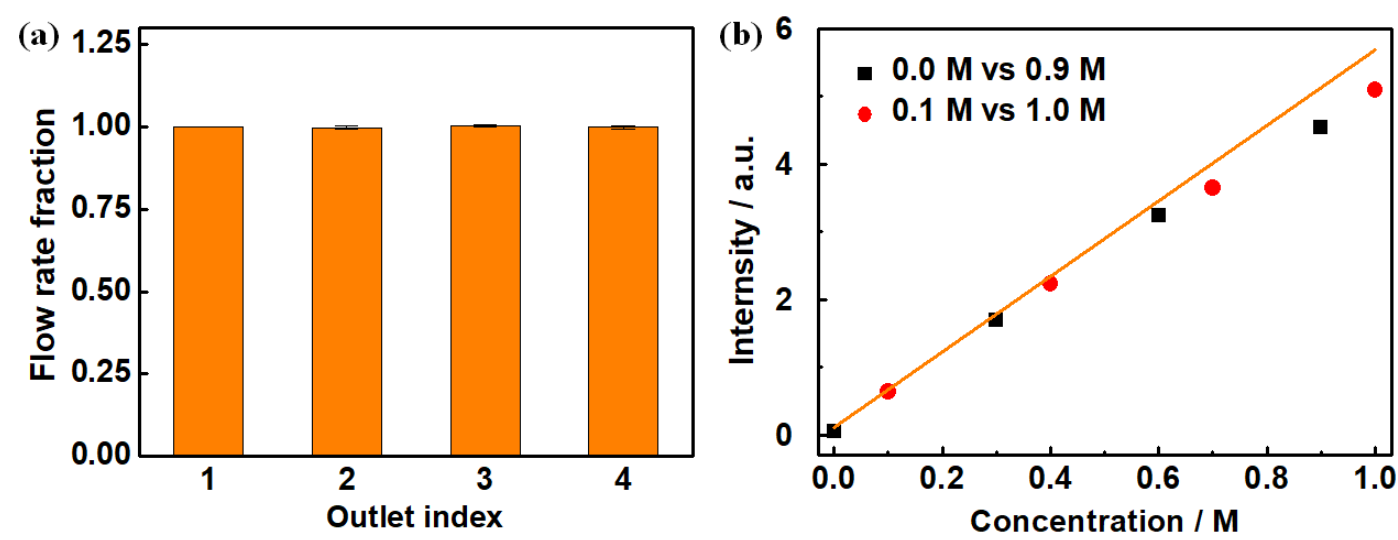

Figure 4
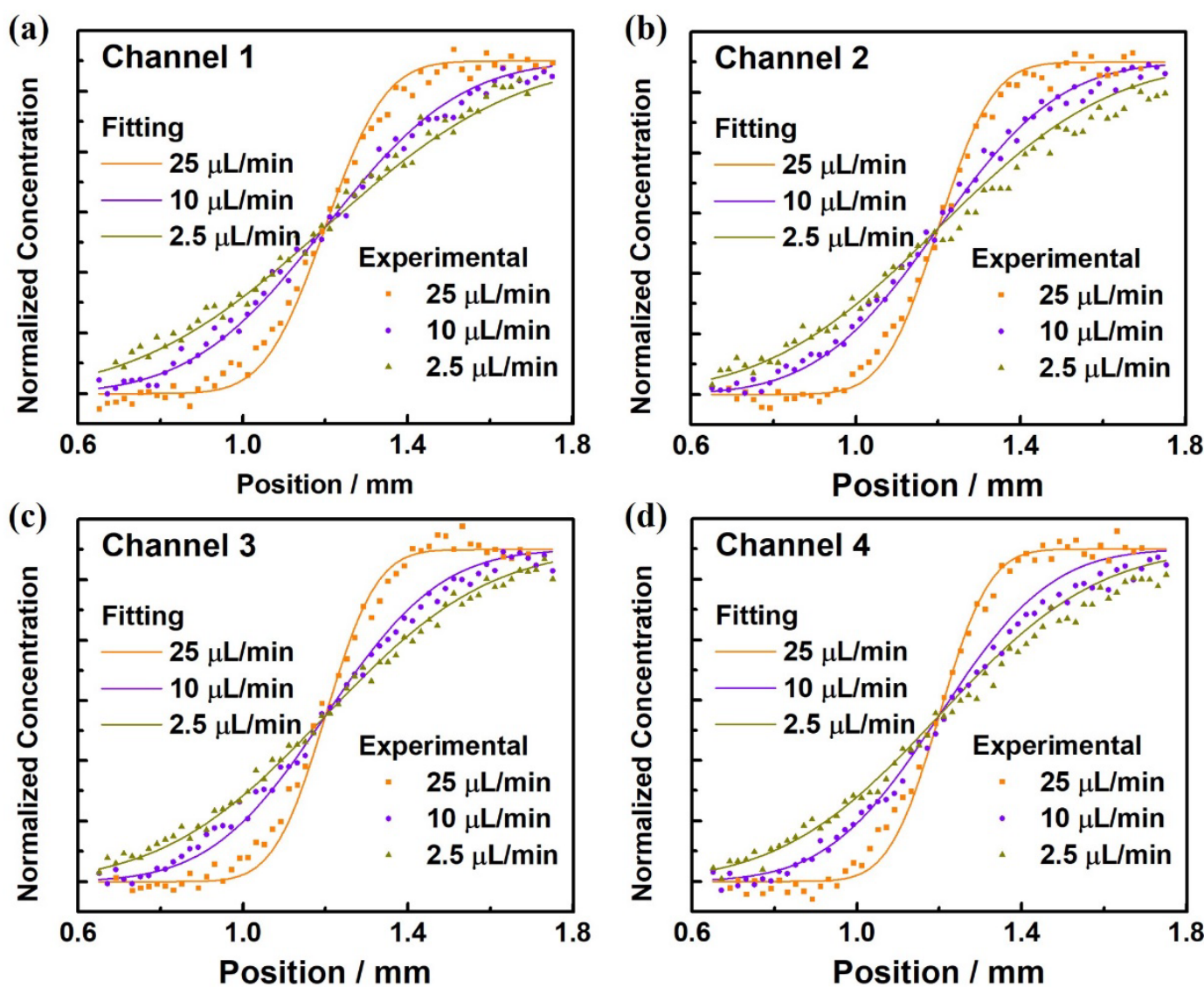